\title{
EUV jets, type III radio bursts and sunspot waves investigated using SDO/AIA observations *
}

\author{
D. E. Innes, R. H. Cameron, and S. K. Solanki
}

\author{
Max-Planck Institut für Sonnensystemforschung, 37191 Katlenburg-Lindau, Germany \\ e-mail: innes@mps.mpg.de
}

Received 13 May 2011 / Accepted 16 June 2011

\begin{abstract}
Context. Quasi-periodic plasma jets are often ejected from the Sun into interplanetary space. The commonly observed signatures are day-long sequences of type III radio bursts.

Aims. The aim is to identify the source of quasi-periodic jets observed on 3 Aug. 2010 in the Sun's corona and in interplanetary space. Methods. Images from the Solar Dynamics Observatory (SDO) at $211 \AA$ are used to identify the solar source of the type III radio bursts seen in WIND/WAVES dynamic spectra. We analyse a $2.5 \mathrm{~h}$ period during which six strong bursts are seen. The radio signals are cross-correlated with emission from extreme ultraviolet (EUV) jets coming from the western side of a sunspot in AR 11092. The jets are further cross-correlated with brightening at a small site on the edge of the sunspot umbra, and the brightening with 3-min sunspot intensity oscillations.

Results. The radio bursts correlate very well with the EUV jets. The EUV jet emission also correlates well with brightening at what looks like their footpoint at the edge of the umbra. The jet emission lags the radio signals and the footpoint brightening by about $30 \mathrm{~s}$ because the EUV jets take time to develop. For 10-15 min after strong EUV jets are ejected, the footpoint brightens at roughly 3 min intervals. In both the EUV images and the extracted light curves, it looks as though the brightening is related to the 3-min sunspot oscillations, although the correlation coefficient is rather low. The only open field near the jets is rooted in the sunspot.

Conclusions. Active region EUV/X-ray jets and interplanetary electron streams originate on the edge of the sunspot umbra. They form along a current sheet between the sunspot open field and closed field connecting to underlying satellite flux. Sunspot running penumbral waves cause roughly 3-min jet footpoint brightening. The relationship between the waves and jets is less clear.
\end{abstract}

Key words. Sun: activity - sunspots - Sun: oscillations - Sun: radio radiation - Sun: UV radiation - magnetic reconnection

\section{Introduction}

$\mathrm{X}$-ray and extreme ultraviolet (EUV) jets in active regions (ARs) are often associated with repetitive type III radio bursts (Chiuderi-Drago et al. 1986; Kundu et al. 1995; Chifor et al. 2008). Recently they have also been linked to ${ }^{3} \mathrm{He}$-rich particle events (Wang et al. 2006; Nitta et al. 2008), and to electron spikes detected at a distance of $1 \mathrm{AU}$ (Klassen et al. 2011).

AR jets are mostly seen on the west of the leading spot (Shimojo et al. 1996). Several were observed by Yohkoh to have a footpoint on the edge of the sunspot umbra (Canfield et al. 1996). Hinode has also observed X-ray jets close to the umbral boundary of a sunspot (Nitta et al. 2008) and a pore (Chifor et al. 2008). Particle acceleration and jet formation is believed to occur at reconnection sites along current sheets that form between flux emerging close to the sunspot and adjacent open field (Shibata et al. 1992; Shimojo et al. 1998), or due to the movement of sunspot satellite flux into a nearby unipolar flux region with the same polarity as the sunspot (Canfield et al. 1996). In most models the sunspot/pore is part of the jet environment but plays only a passive role.

In this Letter we show a connection between the sunspot, the surrounding satellite flux, and EUV jets using high cadence (12 s) Solar Dynamics Observatory (SDO) images from the

\footnotetext{
* Movie is available in electronic form at http://www . aanda.org
}

Atmospheric Imaging Assembly (AIA) and magnetic field observation from the Helioseimic and Magnetic Imager (HMI). We also investigate (with somewhat inconclusive results) the triggering of jets by sunspot waves. The chromospheres of sunspots oscillate with a period of 3 min (e.g. Bogdan 2000). The oscillations were first seen as umbral flashes in the chromosphere (Beckers \& Tallant 1969), and are believed to be magnetoacoustic waves travelling along magnetic field lines (Centeno et al. 2006; Bloomfield et al. 2007). In UV transition region lines both intensity and velocity oscillations are observed across entire sunspot umbrae (Gurman et al. 1982; Brynildsen et al. 1999). The oscillations also extend into coronal loops anchored in the sunspot (De Moortel et al. 2002).

Of special relevance for this Letter is that 3-min oscillations have been observed in microwave flare data (Sych et al. 2009), and this was interpreted as flare triggering by 3-min sunspot waves. Wave-induced reconnection was previously suggested by Ning et al. (2004) when they noticed that in the quiet Sun transition region explosive events occur in bursts and repeat every 35 min. Simulations have shown that the process is viable (Chen \& Priest 2006; Heggland et al. 2009) but because of the multitude of overlapping small-scale activity along the line-of-sight in the quiet Sun it is hard to test. The sunspot-EUV jet relationship discussed here offers a simpler view for assessing the validity of the mechanism. 


\section{Observations and data analysis}

A series of quasi-periodic type III radio bursts was seen in the WIND/WAVES dynamic spectrum from 14:00-24:00 UT on 3 Aug. 2010 (Fig. 1). The source of the bursts was jets from the leading sunspot in AR 11092 (Fig. 2). This region produced a C3 flare on 1 Aug. 2010, and several other sudden heating events in the preceding days as described by Schrijver \& Title (2011). On the 3 Aug. 2010 the active region was near disk center from the Earth perspective so SDO had a face-on view of the sunspot.

SDO has two imaging instruments: AIA and HMI. AIA takes images of the solar disk through 10 different filters, selected to single out specific strong lines in the corona and continuum emission from the lower chromosphere. Photospheric magnetic field, continuum intensity and Doppler velocity images are obtained by the optical instrument, HMI.

The analysis presented here uses primarily full resolution AIA $211 \AA$ images at a cadence of $12 \mathrm{~s}$ and spatial pixel size of $0.6^{\prime \prime}$. We have also made a movie of the jets by combining a short time series of $211 \AA$ and $304 \AA$ with HMI line-of-sight magnetic field images. The AIA are level 1.0 images, obtained via the Virtual Solar Observatory and the HMI are level 1.0, obtained from the German Data Center for SDO. The images have been re-mapped to the same resolution and de-projected to the start time of the observation period. The dominant emission in the $211 \AA$ images is from Fe xIV with a formation temperature $2 \times 10^{6} \mathrm{~K}$. The $304 \AA$ image selects the transition region, He II, line emission with a formation temperature $5 \times 10^{4} \mathrm{~K}$.

The visibility of the EUV jets and the sunspot oscillations is improved in difference images but these are quite confusing when structures are changing rapidly. We reduce the confusion by using base difference images. As a base frame we use the average image over the $2.5 \mathrm{~h}$ period. The difference is the log of the ratio of the image to the base image. This brings out changes in faint structures that may be lost in straight differences.

The radio observations were made with WIND/WAVES (Bougeret et al. 1995). Figure 1 shows the 1 min averaged radio data obtained from the WAVES archive. The data used for cross correlation is the signal integrated between 3 and $8 \mathrm{MHz}$ which is indicated by a bar on the left side of Fig. 1. At higher frequencies there is interference from Earth and at lower frequencies the signal is delayed by more than a min. Type III radio bursts are caused by electron streams propagating through the solar corona and interplanetary space. The distance from the Sun to the radio signal source can be estimated from the frequency and a coronal density model. Leblanc et al. (1998) estimate that $13.6 \mathrm{MHz}$ emission is produced at $2 R_{\odot}$. To determine the electron travel time from the solar surface to $2 R_{\odot}$, we either have to assume an electron energy or velocity. Aschwanden (2002) gives a typical electron velocity $0.14 c$, where $c$ is the speed of light for electrons causing $1 \mathrm{MHz}$ emission. Since these electrons also generate the 13.8 $\mathrm{MHz}$ emission, the travel time for electrons to the site of the observed type IIIs is about $17 \mathrm{~s}$.

\subsection{The origin of the jets}

AR 11092 at the time of the strongest jet is shown in Fig. 2. The jets' dynamics can be seen in the accompanying ${ }^{1}$ which is a three colour composite of the AIA $304 \AA$ and $211 \AA$ filter images and the HMI magnetic field, alongside the base difference $211 \AA$ images. The frame with the strong 16:20 UT jet is shown

\footnotetext{
1 www.mps.mpg.de/data/outgoing/innes/jets/ losb_304_211_rd.gif
}

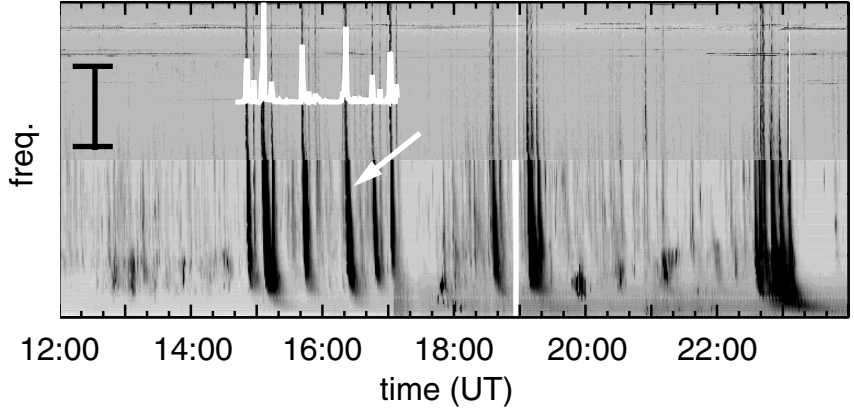

Fig. 1. WIND/WAVES dynamic spectrum from 12:00-24:00 UT on 3 Aug. 2010. The frequency ranges from $20 \mathrm{kHz}$ to $13.6 \mathrm{MHz}$. The black bar on the left indicates the frequency range used to obtain the overplotted white line which is the signal used for cross-correlation. The white arrow points to the radio signal from the 16:20 UT jet shown in Fig. 2.

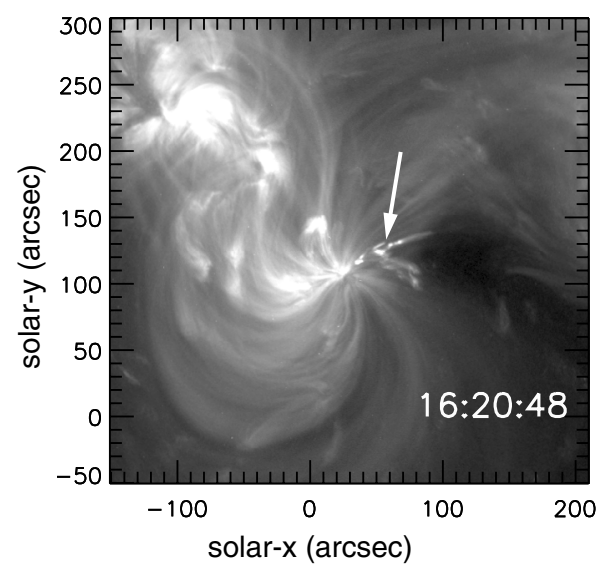

Fig. 2. The SDO/AIA $211 \AA$ image of the active region and one of the jets (white arrow).

in Fig. 3. All the jets are in the same direction and appear just north of some short, low-lying loops that connect the sunspot to a small concentration of satellite flux. This is similar to the jets described by Canfield et al. (1996). In their model the triggering of the jets is caused by the outward motion of the satellite flux driving reconnection at an X-point on the side away from the sunspot. The jets that Canfield et al. (1996) describe were nearly all accompanied by an $\mathrm{H} \alpha$ surge. None of the jets in the movie have $304 \AA$ emission outside the jets which is surprising because surge material should be visible in this filter. It may be that surges present in $\mathrm{H}_{\mathrm{I}}$ are not visible in the $304 \AA$ filter or that surging is common but not fundamental to AR jet dynamics. Another interesting feature of the AIA jets is bright knots of emission moving along the jets, simultaneously in all coronal filters. Further analysis is required to determine whether they are plasmoids formed at jet onset or due to instabilities in the jet.

In the base difference movie the sunspot waves are obvious over the sunspot umbra. Close inspection gives the impression that the waves propagate beyond the umbra along the loops in all directions probably as slow magnetosonic waves (De Moortel et al. 2002). The waves look as though they are propagating across the umbra but the effect is likely due to wave pulses propagating upwards along the cone-shaped umbral field which makes the travel time to the observed layer increase towards the edge of the umbra (Bloomfield et al. 2007). Also conspicuous is recurrent brightening at a small site at the edge of the umbra in 

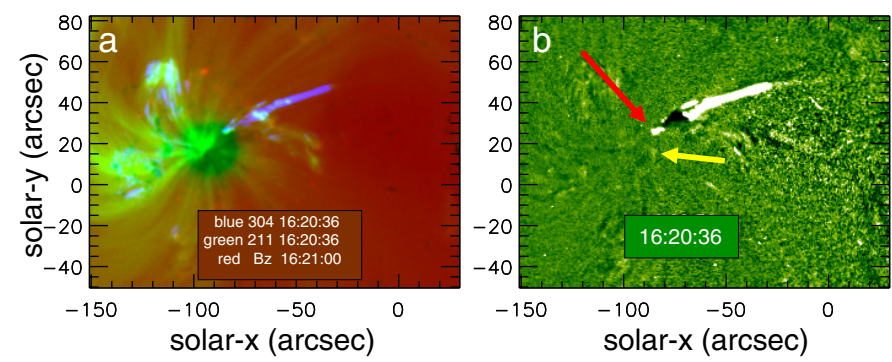

Fig. 3. a) A three colour composite SDO image taken from the accompanying movie. The EUV $304 \AA$ and $211 \AA$ filter images fill the blue and green colours and the red is filled with the line-of-sight magnetic field. b) $211 \AA$ base difference image. The crest of a sunspot wave is indicated with a yellow arrow. The red arrow points to a "footpoint" brightening.

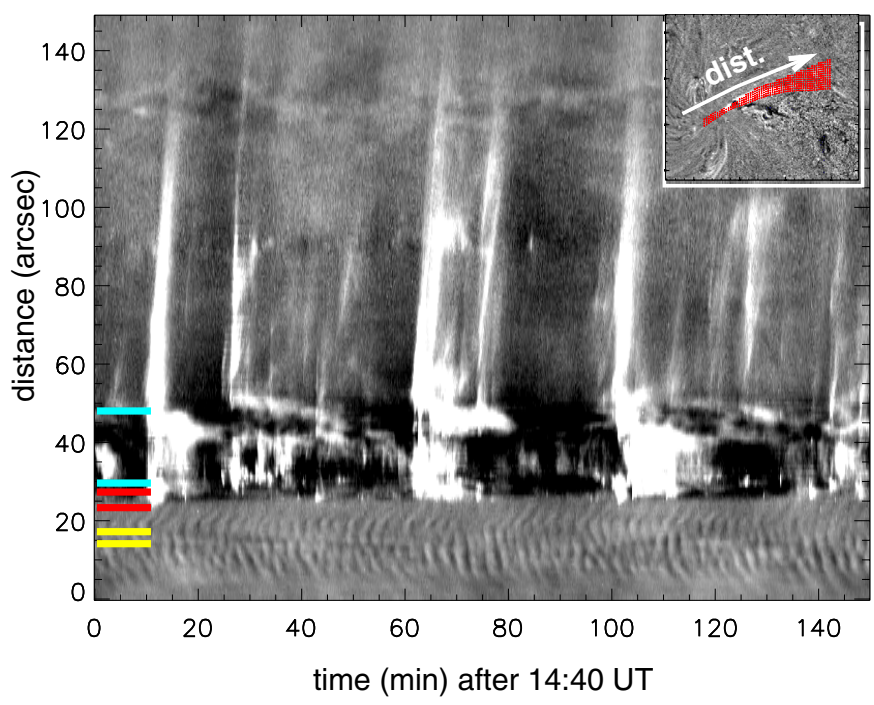

Fig. 4. Time series of $211 \AA$ intensity variations along the red hashed region shown in the inset. At each distance the intensity across the region (perpendicular to the arrow) has been averaged. On the left the yellow and red lines indicate the sunspot and footpoint regions used for cross-correlation. The light blue lines indicate the loop region.

line with the jets. Since this seems to be related but not actually connected to the jets we call this the footpoint.

A time series across the sunspot and along the jet, cutting through the sunspot and footpoint sites used for the cross-correlation in the next section is shown in Fig. 4. The time series is the average intensity over the width of the selected region along the cut as indicated. There are 5-6 strong jets. The strong jets are often followed by a series of weak jets that fade at shorter distances. Each bunch starts with brightening in the region bracketed by red bars. A few minutes later the low-lying loops (light blue region) brighten. The EUV plane-of-sky jet speed ranges from $20-500 \mathrm{~km} \mathrm{~s}^{-1}$ which is typical of X-ray and EUV jets (Shimojo et al. 1996).

\subsection{Timing and cross-correlation}

For the analysis here there are three structures of interest (Fig. 5): the jets that start just beyond the low-lying loops (blue contour), the footpoint on the edge of the umbra that produced recurrent brightenings (red rectangle), and the sunspot umbral oscillations.
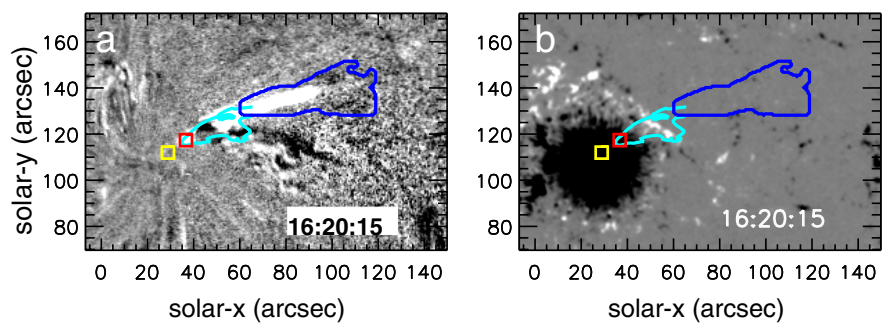

Fig. 5. a) Base difference $211 \AA ̊$ image. b) Line-of-sight magnetic field scaled between $\pm 250 \mathrm{G}$. The yellow rectangles mark the position used for the sunspot intensity oscillation, the red rectangles mark the jet footpoint position, the blue contour outlines the jet region and the light blue the position of the low-lying loops. These regions are used for the cross correlation shown in Fig. 6.

It was noted that the footpoint brightens at the time the EUV jets are observed. An additional question is whether these brightenings and jets are excited by sunspot oscillations. To quantify the relationship we have cross-correlated the intensity of the footpoint with the jet and sunspot at the position marked by the yellow rectangle in Fig. 5. The jet intensity is the intensity integrated within the blue contour.

The temporal evolution of the $211 \AA$ intensity of the different regions is shown in Fig. 6a. The observations have a time cadence of $12 \mathrm{~s}$. The 3-min sunspot oscillations (black line) are very regular throughout the period. Only the six strong jets (blue line) are visible because the weak, diffuse jet signals that are visible in Fig. 4 are lost when integrated over the whole jet region. The footpoint variations also suffer from overlapping loop emission and by our choice of a fixed footpoint site. In spite of these drawbacks, there are distinct short period, roughly 3-min, intensity variations at the footpoint during the strong jet periods. As one can see from the light curves and confirmed by the correlation coefficient, 0.6 , the jet-footpoint correlation is high. The correlation analysis reveals a $30 \mathrm{~s}$ lag of the jet behind the footpoint (Fig. 6c). Making a cross-correlation over the whole $2.5 \mathrm{~h}$ period, yields no correlation between the sunspot waves and the footpoint. But when we focus on two short periods with multiple footpoint brightening (one with a solid and the other with a dotted red line underneath) the correlation coefficient is 0.4 (Fig. 6d).

In Fig. 6b, the intensity variations of the radio signal is compared to the footpoint and jet intensity. The EUV intensities have been averaged over $1 \mathrm{~min}$ intervals to match the radio. Both have a high correlation coefficient (Fig. 6e). The jets seem to lag the radio and the footpoint by $1 \mathrm{~min}$, the cadence of the radio data and consistent with the $30 \mathrm{~s}$ time lag between the jets and the footpoint found with the higher cadence EUV data. This time lag is probably because the jets takes time to develop whereas the other two emissions are compact. Figure 4 suggests that the jet and the footpoint brightening start together.

\section{Discussion}

The SDO images show that the jets causing the type III radio bursts originate near the sunspot umbra. Investigation of the source region has shown a strong correlation between the jet and an EUV brightening at a site on the boundary of the sunspot umbra suggesting that the jets have a footpoint at the edge of the umbra. Starting with strong jets, the footpoint intensity oscillates with a period of about $3 \mathrm{~min}$. The footpoint brightenings 

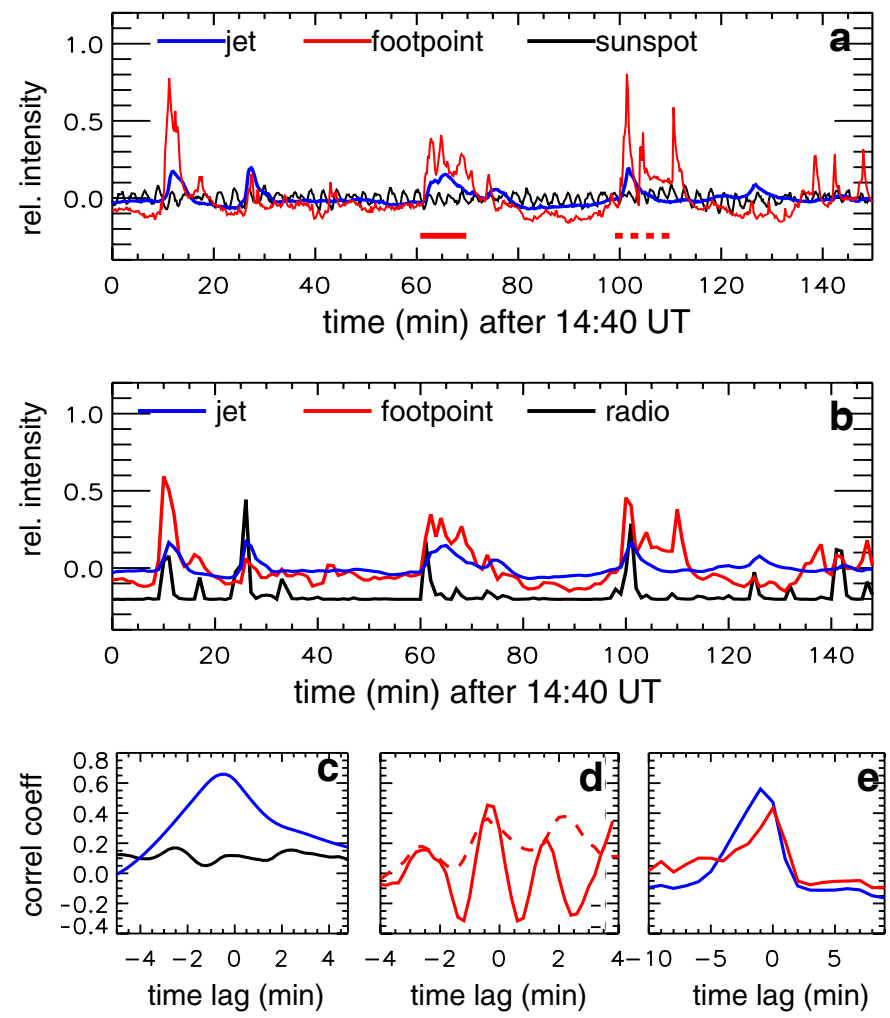

Fig. 6. Light curves of the sunspot, jet and its footpoint: a) $211 \AA$ light curves with $12 \mathrm{~s}$ time cadence of the footpoint, sunspot, and jet; b) $211 \AA$ jet, footpoint, and radio light curves averaged over 1 min; c) cross-correlation between footpoint and the jet (blue) and the footpoint and sunspot (black) over the $2.5 \mathrm{~h}$ period; d) cross-correlation between the footpoint and sunspot during two jet intensive periods as indicated by the solid and dashed red lines in a). e) Cross-correlation of the jet (blue) and footpoint (red) with the radio.

are simultaneous with the radio to within $1 \mathrm{~min}$, and precede the EUV jets' emission by $30 \mathrm{~s}$.

Similar to the X-ray jets studied by Canfield et al. (1996) the jets lie above satellite flux, moving away from the sunspot. Low-lying loops connecting the sunspot to satellite flux often brighten a few min after the jet. We suggest these loops are either newly reconnected loops or loops heated during the reconnection process. There are no noticeable surges in the $304 \AA$ images so the environment is probably not exactly as observed by Canfield et al. (1996).

An important difference between these and the Canfield et al. (1996) jets is the magnetic topology. In these jets there is no open field outside the sunspot beyond the satellite flux. The only open field along which the jets and electron streams can travel is rooted in the sunspot (Fig. 7). The jets (blue contour) appear between the open (black) and closed (red) field regions. There are also no signs of flux emergence in the previous $24 \mathrm{~h}$, so that the emerging flux trigger (Shibata et al. 1992) is also unlikely. Current sheets are however formed along quasi-separatrix layers between the sunspot open and closed field (Demoulin et al. 1996). Similar changes in connectivity were found at the base of outflows on the edge of active regions (Baker et al. 2009). Given the right driving forces, reconnection can be triggered along the current sheet. The fact that we see modulation of the jet footpoint emission with a roughly 3-min period suggests that sunspot oscillations may play a role. On the other hand, the enhanced footpoint emission may be due to the superposition of sunspot wave

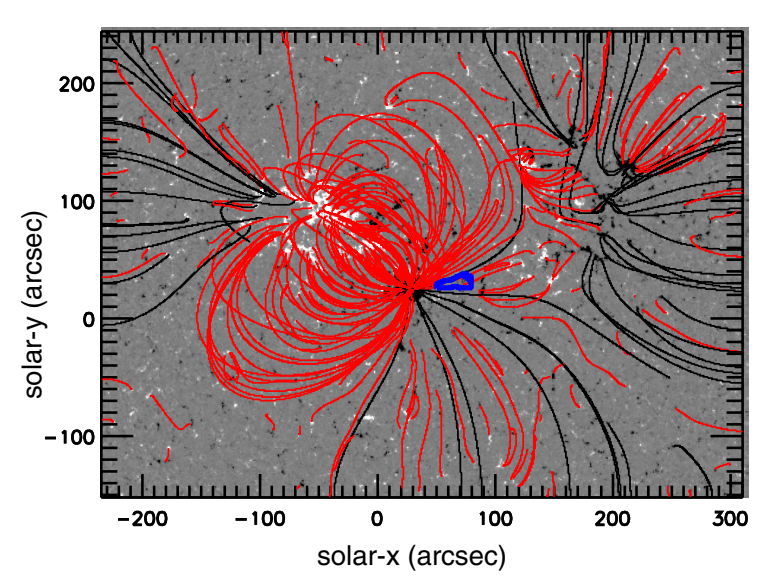

Fig. 7. Potential field extrapolation of AR 11092. Red/black lines are closed/open field lines projected on the line-of-sight magnetic field. The blue contour outlines the jet region.

crests on the reconnection emission so at this stage no firm conclusion can be drawn.

With the AIA/SDO images, we are able to observe jets and their source regions with a temporal and spatial resolution and coverage that allows us to see connections that had been hitherto hidden. In this letter, we have shown that EUV jets have a footpoint on the edge of the sunspot umbra and propose that sunspot coronal currents create the conditions for reconnection and jets.

Acknowledgements. We thank Raphael Attie for the interface to the potential field extrapolation code, and the SDO and WIND/WAVES teams for the data. This work has benefitted from discussions with members of the ISSI, Bern group "Mining SDO data in Europe".

\section{References}

Aschwanden, M. J. 2002, Space Sci. Rev., 101, 1

Baker, D., van Driel-Gesztelyi, L., Mandrini, C. H., Démoulin, P., \& Murray, M. J. 2009, ApJ, 705, 926

Beckers, J. M., \& Tallant, P. E. 1969, Sol. Phys., 7, 351

Bloomfield, D. S., Lagg, A., \& Solanki, S. K. 2007, ApJ, 671, 1005

Bogdan, T. J. 2000, Sol. Phys., 192, 373

Bougeret, J., Kaiser, M. L., Kellogg, P. J., et al. 1995, Space Sci. Rev., 71, 231

Brynildsen, N., Leifsen, T., Kjeldseth-Moe, O., Maltby, P., \& Wilhelm, K. 1999, ApJ, 511, L121

Canfield, R. C., Reardon, K. P., Leka, K. D., et al. 1996, ApJ, 464, 1016

Centeno, R., Collados, M., \& Trujillo Bueno, J. 2006, ApJ, 640, 1153

Chen, P. F., \& Priest, E. R. 2006, Sol. Phys., 238, 313

Chifor, C., Isobe, H., Mason, H. E., et al. 2008, A\&A, 491, 279

Chiuderi-Drago, F., Mein, N., \& Pick, M. 1986, Sol. Phys., 103, 235

De Moortel, I., Ireland, J., Hood, A. W., \& Walsh, R. W. 2002, A\&A, 387, L13

Demoulin, P., Henoux, J. C., Priest, E. R., \& Mandrini, C. H. 1996, A\&A, 308, 643

Gurman, J. B., Leibacher, J. W., Shine, R. A., Woodgate, B. E., \& Henze, W. 1982, ApJ, 253, 939

Heggland, L., De Pontieu, B., \& Hansteen, V. H. 2009, ApJ, 702, 1

Klassen, A., Gómez-Herrero, R., \& Heber, B. 2011, Sol. Phys., in press

Kundu, M. R., Raulin, J. P., Nitta, N., et al. 1995, ApJ, 447, L135

Leblanc, Y., Dulk, G. A., \& Bougeret, J. 1998, Sol. Phys., 183, 165

Ning, Z., Innes, D. E., \& Solanki, S. K. 2004, A\&A, 419, 1141

Nitta, N. V., Mason, G. M., Wiedenbeck, M. E., et al. 2008, ApJ, 675, L125

Schrijver, C. J., \& Title, A. M. 2011, J. Geophys. Res., in press

Shibata, K., Ishido, Y., Acton, L. W., et al. 1992, PASJ, 44, L173

Shimojo, M., Hashimoto, S., Shibata, K., et al. 1996, PASJ, 48, 123

Shimojo, M., Shibata, K., \& Harvey, K. L. 1998, Sol. Phys., 178, 379

Sych, R., Nakariakov, V. M., Karlicky, M., \& Anfinogentov, S. 2009, A\&A, 505, 791

Wang, Y., Pick, M., \& Mason, G. M. 2006, ApJ, 639, 495 